\title{
DETERMINACIÓN DE BTEX EN AIRE AMBIENTE DEL DISTRITO METROPOLITANO DE QUITO MEDIANTE CROMATOGRAFÍA DE GASES CON DETECTOR DE IONIZACIÓN DE LLAMA
}

\author{
DETERMINATION OF BTEX IN AMBIENT AIR OF DISTRITO \\ METROPOLITANO DE QUITO BY GAS CHROMATOGRAPHY \\ WITH FLAME IONIZATION DETECTOR
}

\section{Camila Altamirano $^{1}$ \& Lorena Meneses ${ }^{2}$}

Recibido: 18 septiembre 2017 / Aceptado: 28 noviembre 2017

Palabras claves: BTEX, cromatografía, Quito, ultrasonido, validación.

Key words: BTEX, chromatography, Quito, ultrasonic, validation.

\section{RESUMEN}

La contaminación ambiental puede ser natural o antropogénica, que causa la presencia de determinadas sustancias que interfieren con la salud y el bienestar de los habitantes. En el caso específico del Distrito Metropolitano de Quito (DMQ), los contaminantes se encuentran principalmente en las gasolinas, por esta razón se determinó benceno, tolueno, etilbenceno, $\mathrm{m} / \mathrm{p}$-xileno y o-xileno (BTEX), considerados como contaminantes de origen antropogénico, en el aire

1 Secretaría de Ambiente del Distrito Metropolitano de Quito, Quito, Ecuador (camila.altamirano@ quito.gob.ec)

2 Pontificia Universidad Católica del Ecuador, Facultad de Ciencias Exactas y Naturales, Escuela de Ciencias Químicas, Quito, Ecuador (Immeneses@puce.edu.ec) 
ambiente del DMQ, para identificar las fuentes y períodos de contaminación, con el fin de controlarlas y reducir el impacto ambiental. La determinación de BTEX se realizó mediante el muestreo pasivo junto con una extracción por ultrasonido, utilizando hexano como solvente, para su posterior análisis mediante cromatografía de gases con detector de ionización de Ilama. Las condiciones cromatográficas permitieron la separación, identificación y cuantificación de los compuestos de interés. Se monitoreó BTEX en 43 puntos distribuidos a lo largo y ancho de todo el DMQ con un tiempo de exposición de 30 días. El rango de concentración promedio encontrado durante los meses de octubre 2016 a enero 2017 para benceno fue 0,07 - 13,85 $\mu \mathrm{g} / \mathrm{m}^{3}$; valor que superó la normativa ambiental anual establecida por la Comisión Europea, con un valor de $5 \mu \mathrm{g} / \mathrm{m}^{3}$. El aumento de la concentración de BTEX en el año 2017 frente a los años anteriores, se debe al aumento en la congestión vehicular, así como el uso de combustible en las festividades del mes de diciembre 2016 y enero 2017.

\section{ABSTRACT}

The environmental contamination can be natural or anthropogenic, so this can cause the presence of certain substances that may affect health and comfort of habitants. Because of this, Benzene, Toluene, Ethylbenzene, $\mathrm{m} / \mathrm{p}$-Xylene and oXylene was analyzed in the air of Distrito Metropolitano de Quito (DMQ). In Quito these compounds are present in car fuel, so the pollution sources and period of contamination can be identified and controlled, causing a reduction in the environmental impact. The determination of these compounds was done by passive sampling and then ultrasonic extraction using hexane as solvent for the further analysis in gas chromatography with flame ionization detector. The separation, identification and quantification of the compounds were possible because of the chromatography conditions that were used. BTEX was monitored in 43 places along DMQ, with a period of exposition of 30 days. The mean annual concentration obtained for benzene during October 2016 to January 2017 was $0,07-13,85 \mu \mathrm{g} / \mathrm{m}^{3}$, value that exceed the environmental regulation established by the European Commission, $5 \mu \mathrm{g} / \mathrm{m}^{3}$. The concentration of BTEX have been increasing in 2017 compared to previous years because of vehicular congestions as well as the use of fuel in festivities of December 2016 and January 2017. 


\section{INTRODUCCIÓN}

Los compuestos orgánicos volátiles (COVs), están constituidos fundamentalmente por carbono, y su característica principal es la facilidad de pasar a estado gaseoso debido a que su presión de vapor es baja a 20 ${ }^{\circ} \mathrm{C}$ (Sánchez \& Alcántara, 2008). Además, son compuestos que participan en las reacciones fotoquímicas en la atmósfera y que con los óxidos de nitrógeno en presencia de calor y luz solar producen "esmog" fotoquímico (Secretaría de Medio Ambiente, Recursos Naturales y Pesca, 1998).

Se denomina "esmog" fotoquímico a la contaminación del aire originado por la mezcla de contaminantes de origen primario (óxidos de nitrógeno, hidrocarburos volátiles) con contaminantes secundarios (ozono, radicales, etc.). Los contaminantes secundarios son originados cuando la luz solar incide sobre los contaminantes primarios (Sánchez \& Alcántara, 2008). La manifestación del "esmog" es la formación de una neblina amarillentagrisácea, aerosol de baja humedad y un olor desagradable por la presencia de algunos compuestos gaseosos (Delgado, 2005).
Los compuestos orgánicos volátiles presentes en el aire pueden provenir de fuentes naturales como bosques, zonas húmedas, animales rumiantes, etc., o de fuentes antropogénicas como industria automotriz, uso doméstico o industrial de pinturas y solventes y de reservas de hidrocarburos, donde la fuente antropogénica es la que más aporta a la contaminación del ambiente (Vernier, 1998).

Los principales representantes de los compuestos orgánicos volátiles son Ios BTEX: benceno, tolueno, etilbenceno y xilenos, que se caracterizan por encontrarse en estado gaseoso a temperatura ambiente y por ser insolubles en agua pero solubles en otras sustancias (Ramírez, 2012). Estos compuestos se encuentran en la clasificación de contaminantes peligrosos del aire en la escala nacional de sustancias tóxicas del aire de la Agencia de Protección Ambiental de los Estados Unidos de Norteamérica, debido a que estudios han demostrado que algunos compuestos son cancerígenos o posibles carcinógenos (Agencia de Protección Ambiental de los Estados Unidos de Norteamérica, 2016). 
En Ecuador, el $76 \%$ de la contaminación atmosférica actual proviene del parque automotor, $5 \%$ de industria, 4 \% de generación eléctrica y 15 $\%$ de fuentes naturales, por lo que el monitoreo continuo es necesario, más aún en el Distrito Metropolitano de Quito, debido a las condiciones geográficas que posee, donde la altura a la que se encuentra la ciudad provoca una menor cantidad de oxígeno, lo cual genera que la combustión sea incompleta. Además, las condiciones geográficas que sufre la ciudad al estar rodeada de montañas de la cordillera occidental, provocan que la ventilación sea limitada y como consecuencia, la contaminación se ve atrapada (Marcillo, 2010). Por esta razón, se han establecido diferentes métodos de control para cuantificar estos contaminantes, donde la técnica de análisis químico más usada es desorción térmica aco- plada a cromatografía de gases y detección por espectrofotometría de masas (DT-CG-EM). Sin embargo, la técnica resulta ser costosa, por lo que se han desarrollado nuevas de menor costo, dentro de las cuales se encuentra la cromatografía de gases con detección de ionización de Ilama. El análisis puede desarrollarse in situ mediante uso de bombas de succión, o por captación pasiva donde se usan captadores multilecho (Instituto Nacional de Seguridad e Higiene en el Trabajo, 2013).

Por lo anteriormente expuesto, el objetivo principal de este estudio fue cuantificar benceno, tolueno, etilbenceno y xilenos (BTEX) en aire en el Distrito Metropolitano de Quito mediante cromatografía de gases con detector de ionización de llama (FID) con el fin de determinar la calidad del aire ambiente.

\section{MATERIALES Y MÉTODOS}

\section{Muestreo}

El principio del método es el muestreo pasivo, el cual implica la preparación del muestreador al colocar un sorbente sólido en un contenedor inerte con aberturas de dimensión conocida, que permitan que los vapores de los compuestos orgánicos volátiles (COVs) pasen a través del pasivo a un flujo constante, de manera que puedan quedar adsorbidos 
para su posterior extracción y análisis por cromatografía de gases (EPA, 2014). El muestreador pasivo utilizado fue el de tipo tubo, que consta de un tubo de vidrio relleno con carbón activado que se retiene dentro, al colocar las tapas de difusión, para lo cual se utilizó algodón.

Se determinó que el tiempo de muestreo sería de 30 días y se establecieron los puntos de muestreo, tomando en cuenta la Red de Monitoreo Pasivo de la Secretaría de Ambiente del Distrito Metropolitano de Quito, por lo que se analizaron 43 puntos, que están divididos en las rutas que se indican en la Figura 1. Se muestrearon los meses de octubre, noviembre y diciembre del 2016 y enero del
2017. Se colocó cada mes un muestreador pasivo por punto de muestreo; por lo que se obtuvieron 172 muestras en los 4 meses.

\section{Optimización del método}

Se optimizó el método tomando en consideración la elección del solvente, las condiciones cromatográficas y el tiempo de agitación. Los solventes que se probaron fueron metanol, acetonitrilo y hexano. Las condiciones cromatográficas que variaron fueron la rampa de temperatura, el modo de inyección y el flujo de la columna. Se probaron tres tiempos de agitación, 10, 15 y 20 minutos en baño ultrasonido. 


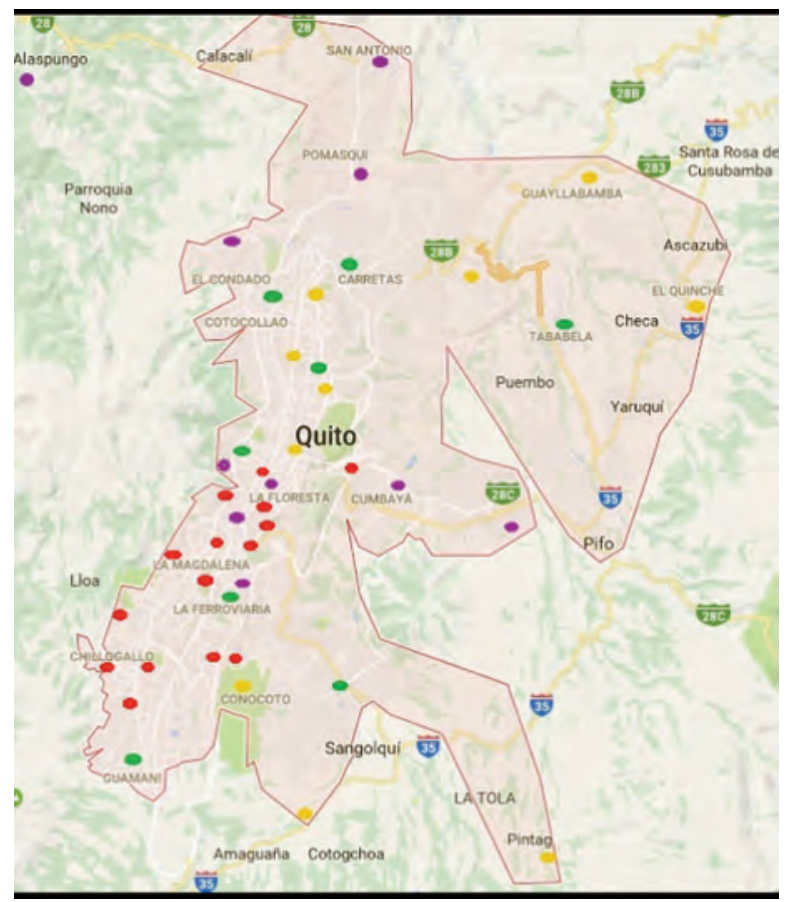

Figura 1. Puntos de muestreo en el Distrito Metropolitano de Quito (DMQ).

Se representan los puntos de muestreo de las rutas: Centro-Sur, Estaciones Fijas, Periféricos y Noroccidente.

Preparación de los muestreadores pasivos

La preparación de los muestreadores pasivos se realizó colocando 0,6 gramos de carbón activado de 80-60 "mesh" en el tubo muestredor para luego ser sellados con papel aluminio y papel parafilm. Al carbón acti- vado se le realizó un acondicionamiento previo, el cual se basó en someter el carbón a $150{ }^{\circ} \mathrm{C}$ durante 48 horas.

Extracción de muestras

La extracción de las muestras se realizó al colocar hexano en el carbón 
activado y someter a agitación de ultrasonido. El extracto se filtró usando un filtro de membrana de celulosa regenerada de 0,45 $\mu \mathrm{m}$. Luego se colocó el extracto en los viales ámbar de $2 \mathrm{~mL}$ para analizar en el cromatógrafo de gases Shimadzu, GC-2014.

\section{Análisis de muestras}

Las muestras se analizaron utilizando un cromatógrafo de gases con detector de ionización de llama. Se utilizó una columna RTX-624 (crossbond 6 $\%$ cianopropilfenil/ $94 \%$ dimetil polisiloxano, de 60 metros, 0,25 $\mathrm{mm}$ de ID, 1,4 um df.). Las temperaturas del inyector y del detector fueron $250{ }^{\circ} \mathrm{C}$ y $300{ }^{\circ} \mathrm{C}$ respectivamente. La rampa de temperatura empezó en $45^{\circ} \mathrm{C}$ por 2 minutos, luego incrementó 10 ${ }^{\circ} \mathrm{C} / \mathrm{min}$ hasta llegar a $150{ }^{\circ} \mathrm{C}$ para luego alcanzar a $220{ }^{\circ} \mathrm{C}$ a $20^{\circ} \mathrm{C} / \mathrm{min}$ y mantenerse ahí por 3 minutos. El gas de arrastre fue helio.

\section{RESULTADOS}

La optimización del método, en especial las condiciones cromatográficas y la elección del solvente, permitió identificar los 5 picos de interés, benceno, tolueno, etilbenceno, $\mathrm{m} / \mathrm{p}$-xileno y o-xileno. Los picos fueron resueltos, estrechos y simétricos de manera que se pudieron cuantificar. En la Figura 2 se puede observar el cromatograma obtenido al muestrear BTEX en la estación Jipijapa en el mes de diciembre utilizando hexano como solvente.

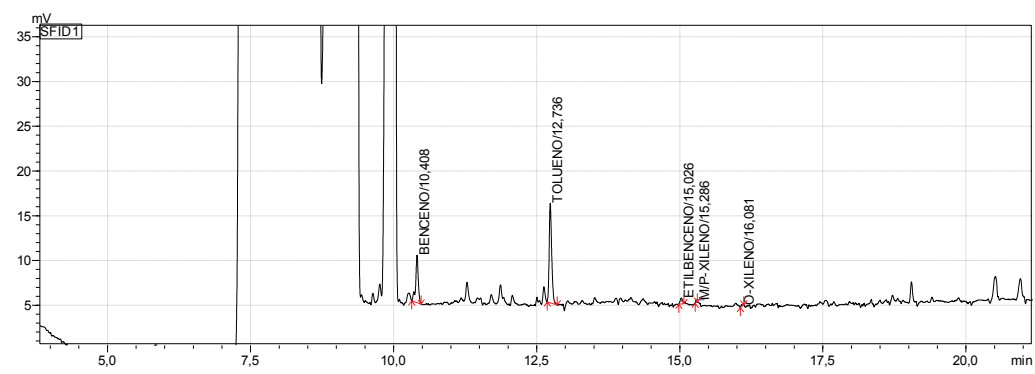

Figura 2. Cromatograma del muestreo de BTEX realizado en la estación Jipijapa en el mes de diciembre de 2016 
Con las condiciones de operación establecidas, se determinó BTEX en el aire ambiente del DMQ en los meses de octubre, noviembre y diciembre de 2016 y enero de 2017. El compuesto de interés fue el benceno, porque está determinado en la Norma de Calidad del Aire Ambiente del Ecuador, Tulsma libro VI, anexo 4, valor tomado de la Comisión Europea con un límite máximo permisible anual de $5 \mu \mathrm{g} / \mathrm{m} 3$ (Comisión Europea, 2016).
Se realizó un promedio de las concentraciones de benceno de los 4 meses de muestreo de manera que se pueda comparar con la normativa ambiental vigente en el DMQ. En la Figura 3, se puede observar las concentraciones de benceno para cada punto de muestreo de los 4 meses de muestreo y en la Figura 4, se pueden observar las concentraciones promedio de benceno de los 4 meses de muestreo.

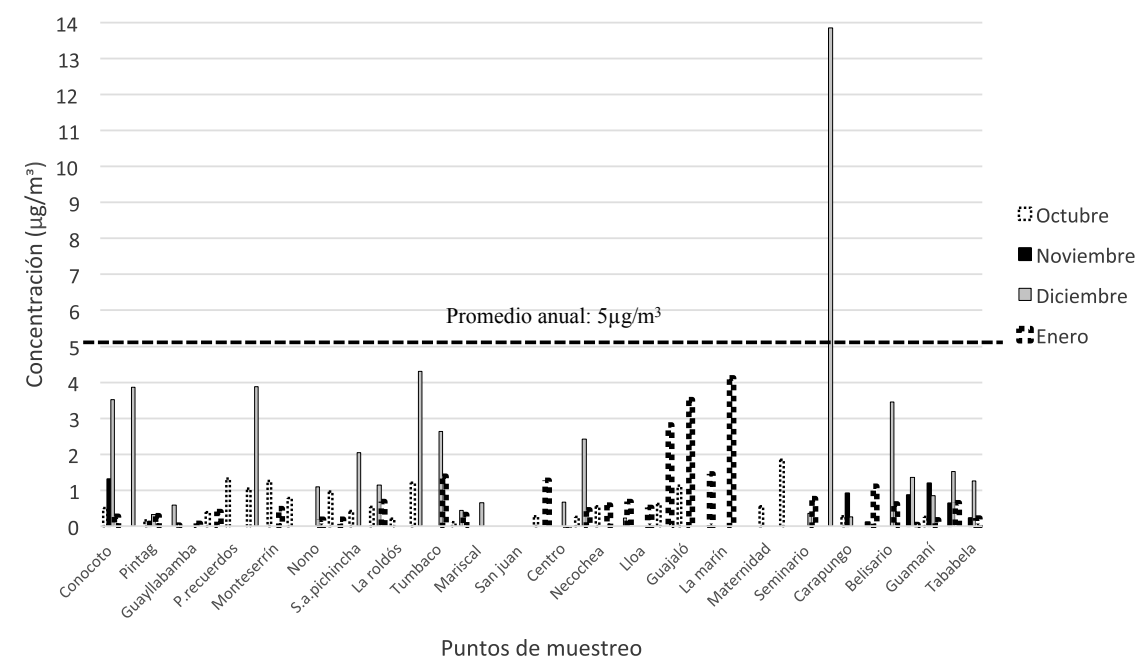

Figura 3. Concentraciones de benceno en los 4 meses de muestreo, en función de los puntos de muestreo 


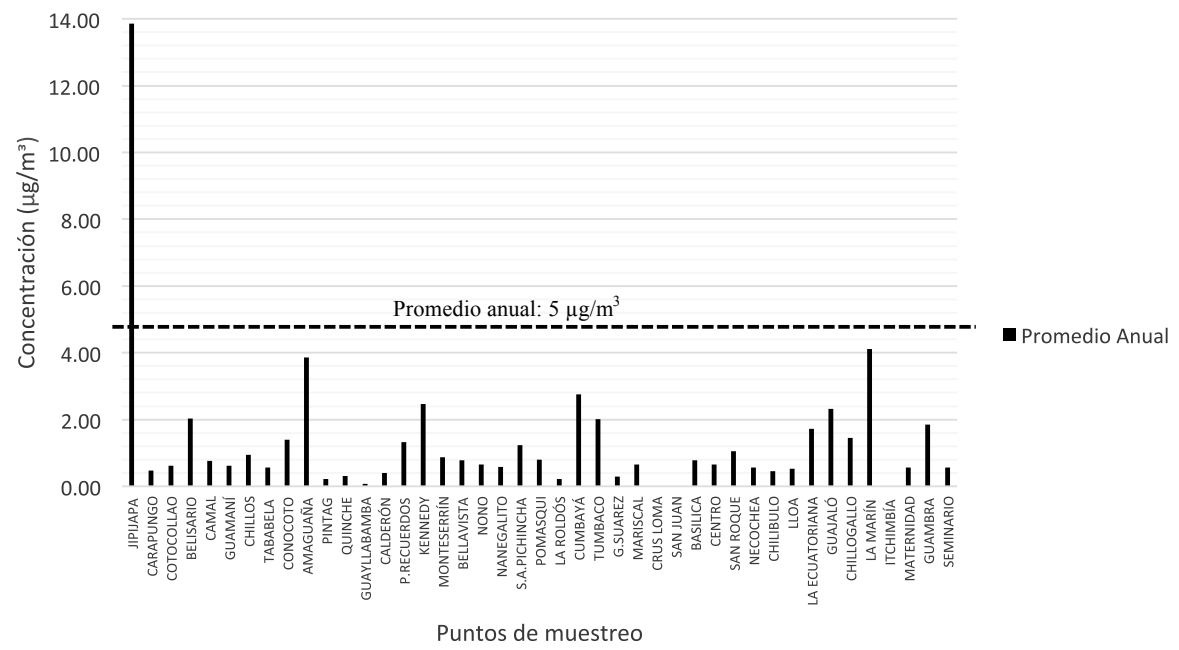

Figura 4. Concentraciones promedio de benceno en los 4 meses de muestreo, en función de los puntos de muestreo

\section{DISCUSIÓN}

La optimización del método permitió identificar los 5 compuestos de interés al seleccionar el solvente de extracción y optimizar las condiciones cromatográficas, porque se evitó la pérdida o coelución de compuestos de interés. Las condiciones cromatográficas permitieron obtener picos estrechos, simétricos y resueltos como se pueden observar en la Figura 2. Además, se controló el tiempo de análisis así como las interferencias posibles en el método.
El monitoreo de BTEX en el Distrito Metropolitano de Quito fue riguroso, ya que los puntos de muestreo cubrieron casi en totalidad a la ciudad, lo que permitió identificar las zonas que se ven más afectadas por estos contaminantes, los puntos de muestreo se pueden observar en la Figura 1. Las concentraciones de benceno, como se puede observar en la Figura 3 , para los meses de octubre, noviembre y enero se encuentra por debajo de la normativa ambiental, 5 $\mu \mathrm{g} / \mathrm{m}^{3}$, a excepción de Jipijapa. El 
punto de muestro localizado en Jipijapa presenta una concentración de $13,85 \mu \mathrm{g} / \mathrm{m}^{3}$. Esto se debe a que en el punto donde se colocó el pasivo se pintaron las cercas, provocando así un aumento repentino de ese punto, porque la pintura generalmente usa ciertos compuestos como solvente, en especial benceno y tolueno. Además, las concentraciones de benceno de los puntos de muestreo Amaguaña, La Kennedy y Cumbayá, presentan valores cercanos a la norma.

La tendencia general en el mes de diciembre es incrementar la concentración del contaminante, esto se debe a varias festividades como las fiestas de Quito, Navidad y Año Nuevo, dónde el tránsito vehicular aumenta. Se queman fuegos pirotécnicos, se lanzan globos de papel, así como muñecos de fin de año, los cuales están conformados por aserrín, el desperdicio de la madera y papel periódico. Para encender estos muñecos y globos se utiliza generalmente gasolina, por lo que se convierten en una fuente de contaminación antropogénica. Al ser festividades, el aumento de venta de cigarrillo aumenta, el cual es otra fuente importante de benceno que puede afectar al aire ambiente del DMQ. Por este motivo, para el mes de diciembre las concentraciones para los BTEX aumentan exponencialmente versus los otros meses de muestreo.

En la Figura 3, en el mes de enero se puede observar que los puntos de muestreo La Marín, Guajaló y La Ecuatoriana presentan valores superiores a $2 \mu \mathrm{g} / \mathrm{m}^{3}$, valores que son altos frente a los otros puntos de muestreo en ese mes. Esto se debe a la gran afluencia vehicular que presentan esas zonas en especial.

En el caso de La Marín, presenta la mayor concentración de benceno, lo que se debe a que en el lugar en el cual se colocó el pasivo hay gran afluencia de buses que provoca un estancamiento vehicular y una mayor emanación de gases.

En el caso de Guajaló, el punto de muestreo se ubicó cerca a una gasolinera y rodeado por el parque industrial del sur de Quito, lo que provoca una mayor concentración de benceno en dichos puntos.

Hay ciertas estaciones que poseen concentraciones de benceno cerca- 
nas a la normativa, y el punto de muestreo Jipijapa la supera, por lo que es necesario considerar la incertidumbre que aporta el muestreo pasivo, que puede afectar a que dichos valores varíen.

En la Figura 4, se puede observar que la tendencia general de benceno en aire ambiente en el DMQ es de 2 $\mu \mathrm{g} / \mathrm{m}^{3}$, donde ciertos puntos obtienen concentraciones cercanas a la normativa. Además, se puede observar que el muestreo de 4 meses no fue suficiente para determinar cumplimiento o incumplimiento con la normativa ambiental, debido a la presencia de un episodio puntual de contaminación como lo fue el mes de diciembre de 2016. Se recomendaría muestrear todo el año para tomar en consideración todas las variables que afectan al contaminante, es especial abarcar la variabilidad de las condiciones atmosféricas.

\section{CONCLUSIONES}

La elección de hexano como solvente, la optimización de las condiciones cromatográficas y establecer un tiempo de extracción de 20 minutos, permitió la identificación de los 5 compuestos de interés, benceno, tolueno, etilbenceno, $\mathrm{m} / \mathrm{p}$-xileno y $\mathrm{o}$ xileno para su posterior cuantificación.

El aumento de la concentración de benceno en el mes de diciembre se debe a los días festivos que se presentan, como fiestas de Quito, Navidad, y Año Nuevo.
El punto de muestreo que sobrepasó la normativa ambiental fue Jipijapa, debido a la captación del contaminante por usar pinturas cercanas al muestreador pasivo.

Los puntos de muestreo de La Marín, Guajaló y La Ecuatoriana presentan valores cercanos a la norma, esto se debe a la congestión vehicular que existe en estos puntos y la existencia de gasolineras cercanas que provocan un aumento en los contaminantes. 


\section{LISTA DE REFERENCIAS}

Agencia de Protección Ambiental de los Estados Unidos de Norteamérica (EPA). (2014). Passive Samplers for Investigations of Air Quality: Method Description, Implementation, and Comparison to Alternative Sampling Methods. Estados Unidos de Norteamérica.

Agencia de Protección Ambiental de los Estados Unidos de Norteamérica (EPA). (2016). TRI-Listed Chemicals Toxics Release Inventory (TRI) Program. Recuperado de: https://www.epa.gov/toxics-release-inventory-tri-program/tri-listed-chemicals

Comisión Europea (2016). Air Quality Standards. Recuperado de: http://ec.europa.eu/environment/air/quality/standards.htm

Delgado, J. (2005). Validación e implementación de técnicas de captación pasiva para el estudio de los niveles y efectos de ozono troposférico y dióxido de nitrógeno en un área costera mediterránea (Disertación de doctorado). Universitat Jaume I, Castellón de la Plana, España.

Instituto Nacional de Seguridad e Higiene en el Trabajo. (2013) Compuestos orgánicos volátiles: determinación por captación en tubos multilecho y análisis DT-CG-EM. Recuperado de http://upcommons.upc.edu/bitstream/handle/2117/21016/ntp978.pdf

Marcillo, A. (2010). Desarrollo de una técnica de muestreo pasivo y análisis por HPLC (UV-VIS) para la determinación de aldehídos en aire del Distrito Metropolitano de Quito (Disertación de pregrado). Pontificia Universidad Católica del Ecuador, Quito, Ecuador.

Ramírez, V. (2012). Cuantificación de compuestos aromáticos (BTEX) en las emisiones gaseosas de fuentes móviles terrestres de gasolina en el Distrito Metropolitano de Quito (Disertación de pregrado). Universidad Central del Ecuador, Quito, Ecuador.

Sánchez, J. y Alcántara, A. (2008). Compuestos orgánicos volátiles en el medio ambiente. Madrid. 
Secretaría de Medio Ambiente, Recursos Naturales y Pesca. (1998). Norma Oficial Mexicana NOM-123-ECOL-1998, que establece el contenido máximo permisible de compuestos orgánicos volátiles (COVs), en la fabricación de pinturas de secado al aire base disolvente para uso doméstico y los procedimientos para la determinación del contenido de los mismos en pinturas y recubrimientos. Recuperado de http://www.profepa.gob.mx/innovaportal/file/1282/1/nom-123-semarnat-1998.pdf

Vernier, J. (1998). El medio Ambiente. México: Publicaciones Cruz O., S.A. 
